\title{
Analysis of stationary random responses for non-parametric probabilistic systems
}

\author{
Y. Zhao ${ }^{\text {a }}$, Y. H. Zhang ${ }^{\text {a,* }}$, J. H. Lin ${ }^{\text {a }}$, W. P. Howson ${ }^{\mathrm{b}}$ and F. W. Williams ${ }^{\mathrm{b}}$ \\ ${ }^{a}$ State Key Laboratory of Structural Analysis for Industrial Equipment, Faculty of Vehicle Engineering and \\ Mechanics, Department of Engineering Mechanics, Dalian University of Technology, Dalian 116023, P.R. China \\ ${ }^{\mathrm{b}}$ Cardiff School of Engineering, Cardiff University, Cardiff CF24 3AA, Wales, UK
}

\begin{abstract}
The move from conceptual design, through fabrication to observation and measurement on the resulting physical structure is fraught with uncertainty. This, together with the necessary simplifications inherent when using the finite element technique, makes the development of a predictive model for the physical structure sufficiently approximate that the use of random structural models is often to be preferred. In this paper, the random uncertainties of the mass, damping and stiffness matrices in a finite element model are replaced by random matrices, and a highly efficient pseudo excitation method for the dynamic response analysis of non-parametric probability systems subjected to stationary random loads is developed. A numerical example shows that the dynamic responses calculated using a conventional (mean) finite element model may be quite different from those based on a random matrix model. For precise fabrication, the uncertainties of models cannot be ignored and the proposed method should be useful in the analysis of such problems.
\end{abstract}

\section{Introduction}

Practical structures cannot be constructed as accurately as they were designed and perfect observation or measurement of an existing structure is also impossible. Therefore the mean-valued model of a practical structure, as is commonly used in conventional FE analysis, is only an approximation of the ideal or theoretical structure. In order to improve the reliability of predictions of structural responses, it is natural and reasonable to use stochastic models [1, 2]. To date, such stochastic factors have been restricted to specific applications, e.g. to either the structural model or the excitations, but not both at the same time. Establishing a methodology for incorporating both the structural model and its excitations into a single model and solving the resulting model numerically is developed herein.

In general, the uncertainty associated with a practical structure consists of uncertainty of the data and uncertainty of the model $[3,4]$. Uncertainty of the data means uncertainty about some of the structural parameters, e.g. geometric sizes, Young's modulus, material density, etc., which can all be depicted by means of appropriate probabilistic models. Data uncertainties are usually modeled using parametric probabilistic models, for which there are some very efficient tools available e.g. Structural reliability methods [5-7] and stochastic FEM [1,8-11]. When parametric probabilistic models are used to depict the uncertainties of dynamic systems, it is necessary to know exactly the parameters which are uncertain, as well as their probabilistic distribution characteristics. If there are too many uncertain parameters, particularly when such uncertain parameters are unknown, the parametric probabilistic models cannot be used.

Uncertainty of the model is caused because it is unlikely that all details of the structure are known, either accurately or at all. As a result, some simplifications must be made when modeling the structure and they are non-parametric

${ }^{*}$ Corresponding author: Dr. Y. H. Zhang, State Key Laboratory of Structural Analysis for Industrial Equipment, Department of Engineering Mechanics, Dalian University of Technology, Dalian 116023, P. R. China. Tel.: +86 411 84706337; Fax: +86 411 84708400; E-mail: zhangyh@dlut.edu.cn. 
and so cannot be dealt with by means of probabilistic parameter models. Hence the theory of random matrices has been considered a good alternative choice [12] and so refs $[3,4,13,14]$ investigated the dynamic responses of such uncertainty models when subjected to deterministic loads. More recently, the Wishart random matrix simulation algorithm, which is both efficient and computationally simple, has been used to quantify the uncertainty in the mass, stiffness and damping matrices of a cantilever plate. These values were then used successfully to predict the response of the plate over a wide range of frequencies $[15,16]$. For random matrix models of general dynamic systems, it is not necessary to identify the uncertain parameters for the FE model, so this method is sometimes termed the non-parametric probabilistic model.

When an uncertain model is used, the loads are usually assumed to be deterministic, even though in practice they are random. The present paper has overcome this shortcoming by extending these single stochastic problems into double-stochastic ones, so that not only is the structural model uncertain, but so are the loads, which can also be random e.g. earthquakes. For the methods proposed, the uncertainties of the stiffness, damping and mass matrices are modeled using the theory of random matrices and the uncertainty of the seismic loads is processed by means of the Pseudo-Excitation Method (PEM) [17-19]. Numerical examples show the correctness and effectiveness of the proposed method.

\section{Concept of a random matrix}

From the viewpoint of mathematical statistics, an accurate description of the system microstate, which would be impossible to achieve, is not necessary. Instead the concept of "ensemble" is of more significance. Herein, ensemble means a large set of completely identical systems satisfying certain macroscopic constraint conditions. The microstate of the system now has a certain statistical distribution that enables the required macrostate to be given by the ensemble mean values of the corresponding quantities. FE models of structural dynamic systems are usually expressed in terms of matrices and therefore the statistical ensemble, which represents stochastic mechanical characteristics, can be described by means of random matrix theory.

\subsection{Macroscopic constraint conditions of random matrices}

Let $\mathbf{A}$ be an $n \times n$ positive-definite, symmetric random matrix. In order to deduce its probability density function $p_{A}$, the available information yields only some of its macroscopic properties, i.e. the macroscopic constraint conditions. In general, the expectation constraints $\overline{\mathbf{A}}=E[\mathbf{A}]$ form a frequently used representation of the macroscopic constraint conditions. An alternative is to use $E[\ln (\operatorname{det}[\mathbf{G}])]=v,|v|<+\infty$. By using the integral characteristic of $p_{A}$, the general expression for the macroscopic constraint conditions of the random matrix $\mathbf{A}$ can be written as

$$
\begin{aligned}
& \int p_{A}(\mathbf{A}) \tilde{d} A=1 \\
& \int \mathbf{A} p_{A}(\mathbf{A}) \tilde{d} A=\overline{\mathbf{A}} \\
& \int \ln (\operatorname{det} \mathbf{A}) p_{A}(\mathbf{A}) \tilde{d} A=v, \quad|v|<+\infty
\end{aligned}
$$

in which $\tilde{d} A$ is the volume element, such that

$$
\tilde{d} A=2^{n(n-1) / 4} \prod_{1 \leqslant i \leqslant j \leqslant n} d \mathbf{A}_{i j}
$$

while matrix $\overline{\mathbf{A}}$ is the expectation of random matrix $\mathbf{A}$. 


\subsection{The mathematical expression for the probability density function of the random matrix}

The probabilistic density function of a random matrix in the probabilistic space can be determined by using the maximum entropy principle under macroscopic constraint conditions (1)-(3). In general, such problems can only be solved by means of numerical methods, which enable the maxima of multivariate functions to be calculated. However, for some important cases, the problem can be solved analytically or by simplification to an algebraic equation. For the special case when the macroscopic constraint conditions are given by means of the expectations, the probabilistic density function can be derived from the variational method, in combination with Lagrange multipliers or Euler's equation [20]. The probability density function of the random matrix can be expressed as [13]

$$
p_{A}(\mathbf{A})=c_{A} \times(\operatorname{det} \mathbf{A})^{\lambda_{A}-1} \times \exp \left(-\frac{\left(n-1+2 \lambda_{A}\right)}{2} \operatorname{tr}\left\{\overline{\mathbf{A}}^{-1}, \mathbf{A}\right\}\right)
$$

in which $\lambda_{A}>0\left(1-\lambda_{A}\right.$ is the Lagrange multiplier corresponding to the constraints defined by Eq. (3)) and

$$
c_{A}=\frac{(2 \pi)^{-n(n-1) / 4}\left(\frac{n-1+2 \lambda_{A}}{2}\right)^{n\left(n-1+2 \lambda_{A}\right) / 2}}{\left\{\prod_{l=1}^{n} \Gamma\left(\frac{n-l+2 \lambda_{A}}{2}\right)\right\}(\operatorname{det} \overline{\mathbf{A}})^{\left(n-1+2 \lambda_{A}\right) / 2}}
$$

in which $\Gamma(x)=\int_{0}^{+\infty} t^{x-1} e^{-x} d t(x>0)$ is the gamma function. Equation (6) relates to the multivariate gamma function and more details can be found in references [21,22].

\subsection{Monte-Carlo simulation of random matrices}

Cholesky factorization of $\overline{\mathbf{A}}$ gives

$$
\overline{\mathbf{A}}=\overline{\mathbf{L}}_{A}^{T} \overline{\mathbf{L}}_{A}
$$

in which $\overline{\mathbf{L}}_{A}$ is an upper triangular matrix. Consequently the random matrix $\mathbf{A}$ can be written as

$$
\mathbf{A}=\overline{\mathbf{L}}_{A}^{T} \mathbf{G} \overline{\mathbf{L}}_{A}
$$

in which $\mathbf{G}$ is a random matrix with its mean value being an $n \times n$ unit matrix, i.e. $\overline{\mathbf{G}}=E[\mathbf{G}]=\mathbf{I}$.

Thus, in order to generate the random matrix $\mathbf{A}$, it is only necessary to carry out a Monte Carlo numerical simulation for random matrix $\mathbf{G}$, by following the steps below [13,14]:

(1) Factorize the random matrix $\mathbf{G}$ into $\mathbf{G}=\mathbf{L}^{T} \mathbf{L}$, in which $\mathbf{L}$ is an upper triangular random matrix with elements $L_{i j}(i \leqslant j)$ which are mutually independent.

(2) When $i<j, L_{i j}$ is a real valued random variable, which can be expressed as $L_{i j}=\sigma_{n} U_{i j}$, in which $\sigma_{n}=\delta(n+1)^{-1 / 2}(\delta$ is the dispersion parameter that controls the deviation of a random matrix $)$ and $U_{i j}$ is a real valued Gaussian random variable with zero mean value and unit variance.

(3) When $i=j$, the real valued random variable $L_{i j}$ can be expressed as $L_{i j}=\sigma_{n} \sqrt{2 V_{i}}$, where $V_{i}$ is a positive valued gamma random variable, with probability density function

$$
p_{v_{i}}(v)=\frac{1}{\Gamma\left((n+1) /\left(2 \delta^{2}\right)+(1-i) / 2\right)} \times v^{(n+1) /\left(\left(2 \delta^{2}\right)-(1+i) / 2\right)} e^{-v}
$$

\section{Stationary random responses of non-parametric probabilistic systems}

In this section, the non-parametric uncertainties of the system are modeled by random matrix theory, and the system is assumed to be subjected to given stationary random loads. The random responses are computed and investigated by means of the highly efficient PEM (or fast-CQC method [17-19]). 


\subsection{Fast-CQC random vibration computation for non-parametric probabilistic systems}

When the uncertainties of mass, damping and stiffness matrices are modeled by random matrices, the equations of motion of structures subjected to stationary random excitations have the form [23]

$$
\mathbf{M} \ddot{\mathbf{y}}(t)+\mathbf{C} \dot{\mathbf{y}}(t)+\mathbf{K y}(t)=\mathbf{p} w(t)
$$

in which $\mathbf{M}, \mathbf{C}$ and $\mathbf{K}$ are $n \times n$ symmetric positive-definite random matrices; $\mathbf{p}$ is a constant vector and $w(t)$ is a stationary random process with known PSD matrix $S_{w w}(\omega)$.

Corresponding to Eq. (10), the motion equations for the mean-valued FE model are

$$
\overline{\mathbf{M}} \ddot{\mathbf{y}}(t)+\overline{\mathbf{C}} \dot{\mathbf{y}}(t)+\overline{\mathbf{K}} \mathbf{y}(t)=\mathbf{p} w(t)
$$

in which $\overline{\mathbf{M}}, \overline{\mathbf{C}}$ and $\overline{\mathbf{K}}$ are the mean-valued matrices of the random matrices $\mathbf{M}, \mathbf{C}$ and $\mathbf{K}$.

It is assumed that the random matrices $\mathbf{M}, \mathbf{C}$ and $\mathbf{K}$ are independent of one another and also of the random process $w(t)$. They can then be expressed by the corresponding probability matrix models as

$$
\begin{aligned}
& \mathbf{M}=\overline{\mathbf{L}}_{M}^{T} \mathbf{G}_{M} \overline{\mathbf{L}}_{M} \\
& \mathbf{C}=\overline{\mathbf{L}}_{C}^{T} \mathbf{G}_{C} \overline{\mathbf{L}}_{C} \\
& \mathbf{K}=\overline{\mathbf{L}}_{K}^{T} \mathbf{G}_{K} \overline{\mathbf{L}}_{K}
\end{aligned}
$$

in which the upper triangular random matrices $\overline{\mathbf{L}}_{M}, \overline{\mathbf{L}}_{C}$ and $\overline{\mathbf{L}}_{K}$ correspond to Cholesky factorization of the mean matrices $\overline{\mathbf{M}}, \overline{\mathbf{C}}$ and $\overline{\mathbf{K}}$, so that $\overline{\mathbf{M}}=\overline{\mathbf{L}}_{M}^{T} \overline{\mathbf{L}}_{M}, \overline{\mathbf{C}}=\overline{\mathbf{L}}_{C}^{T} \overline{\mathbf{L}}_{C}$ and $\overline{\mathbf{K}}=\overline{\mathbf{L}}_{K}^{T} \overline{\mathbf{L}}_{K}$. The random matrices $\mathbf{G}_{M}, \mathbf{G}_{C}$ and $\mathbf{G}_{K}$ can be generated by following the procedure set out in Section 2.3.

For the stationary random vibration analyses of non-parametric probability systems, the solution of Eq. (10) can be expressed by Duhamel integration as

$$
\mathbf{y}(t)=\int_{0}^{t} \mathbf{h}(t, \tau) \mathbf{p} w(t) d \tau
$$

in which $\mathbf{h}(t, \tau)$ is the impulse response matrix at instant $\tau$ and $w(t)$ is a stationary random process that is statistically uncorrelated with the uncertainties in the system. The covariance matrix of any arbitrary response vector $\mathbf{y}$ is

$$
\mathbf{R}_{y y}(t)=E\left[\mathbf{y y}^{T}\right]=\int_{0}^{t} \int_{0}^{t} \mathbf{h}\left(t, \tau_{1}\right) E\left[\left(\mathbf{p} w\left(\tau_{1}\right)\right)\left(\mathbf{p} w\left(\tau_{2}\right)\right)^{T}\right] \mathbf{h}^{T}\left(t, \tau_{2}\right) d \tau_{1} d \tau_{2}
$$

The solution of Eq. (16) is usually achieved by reducing the equation to a double series summation, which is extremely demanding in both time and effort. In this paper, the PEM or fast-CQC method [17-19] is used instead.

Let the pseudo ground acceleration be

$$
w(t)=\sqrt{S_{w w}(\omega)} e^{i \omega t}
$$

Substituting into Eq. (10) yields the harmonic equation

$$
\mathbf{M} \ddot{\tilde{\mathbf{y}}}(t)+\mathbf{C} \dot{\tilde{\mathbf{y}}}(t)+\mathbf{K} \tilde{\mathbf{y}}(t)=\mathbf{p} \sqrt{S_{w w}(\omega)} e^{i \omega t}
$$

This means that the stationary random vibration analysis has been transformed into a determinate harmonic vibration analysis. The pseudo harmonic responses of the system thus obtained can be expressed in the form

$$
\tilde{\mathbf{y}}=\hat{\mathbf{y}} e^{i \omega t}
$$

The corresponding PSD matrix $\mathbf{S}_{y y}$ can then be computed using the pseudo harmonic responses [18,19] as

$$
\mathbf{S}_{y y}(\omega ; \mathbf{M}, \mathbf{C}, \mathbf{K})=\tilde{\mathbf{y}}^{*} \tilde{\mathbf{y}}^{T}
$$

while the corresponding variance is

$$
\sigma_{y}^{2}(\mathbf{M}, \mathbf{C}, \mathbf{K})=2 \int_{0}^{\infty} S_{y y}(\omega ; \mathbf{M}, \mathbf{C}, \mathbf{K}) d \omega
$$




\subsection{Convergence analysis of stationary random response computations for non-parametric probabilistic systems}

In order to investigate the convergence of non-parametric probabilistic systems subjected to stationary random loads, let

$$
Q=\left\|\sigma_{y}^{2}\right\|_{2}
$$

in which $\sigma_{y}^{2}$ is the variance response and can be computed using Eq. (21).

For uncertain non-parametric probabilistic systems, the norm vector $Q$ of the stationary random variance response vector, which is real valued, has the form

$$
E[Q] \simeq \frac{1}{n_{s}} \sum_{i=1}^{n_{s}} Q_{i}
$$

\subsection{Confidence region of stationary random responses for non-parametric probabilistic systems}

For a non-parametric probabilistic system subjected to a given stationary random load, let $\theta$ be an arbitrary random response. For a given confidence coefficient $\gamma$, the confidence region of random variable $\theta$ can be expressed as [20]

$$
P\left\{\theta_{1}<\theta<\theta_{2}\right\}>\gamma
$$

in which

$$
\theta_{1}=\bar{\theta}-\frac{\sigma}{\sqrt{1-\gamma}} \theta_{2}=\bar{\theta}+\frac{\sigma}{\sqrt{1-\gamma}}
$$

where $\bar{\theta}$ is the expected value of $\theta$ and $\sigma$ is its standard deviation.

In general, the standard deviation can be estimated by using Monte Carlo numerical simulation, via the equation

$$
\sigma^{2} \simeq \hat{\sigma}^{2}=\frac{1}{n_{s}-1} \sum_{i=1}^{n_{s}}\left(\theta_{i}-\bar{\theta}\right)^{2}
$$

in which $\hat{\sigma}^{2}$ is the unbiased estimator of $\sigma^{2}$.

\section{Numerical example}

The 72-bar spatial truss of Fig. 1, which is subjected to seismic random loading, is investigated. The parameters of all bars are: mass density $7.849 \times 10^{3} \mathrm{~kg} / \mathrm{m}^{3}$, Young's Modulus $2.058 \times 10^{11} \mathrm{~N} / \mathrm{m}^{2}$, sectional area $6.645 \times 10^{-3} \mathrm{~m}^{2}$. Nodes 17-20 each have a lumped mass of $5 \mathrm{~kg}$ attached to them.

For the mean valued model (MVM), the Rayleigh damping matrix $\overline{\mathbf{C}}=0.02 \overline{\mathbf{K}}$ is adopted. For the non-parametric probabilistic model (NPM), it is only necessary that the mean values of the random damping matrix $\mathbf{C}$ and the random stiffness matrix $\mathbf{K}$ meet the condition $\overline{\mathbf{C}}=0.02 \overline{\mathbf{K}}$. However, $\mathbf{C}$ and $\mathbf{K}$ are uncorrelated.

The structure is subjected to a horizontal seismic acceleration in the y-direction, which is regarded as a stationary Gaussian random process. This seismic acceleration has a Kanai-Tajimi filtered white-noise spectrum with the form

$$
S_{w w}(\omega)=\frac{1+4\left(\zeta_{g} \omega / \omega_{g}\right)^{2}}{\left(1-\omega^{2} / \omega_{g}^{2}\right)^{2}+4\left(\zeta_{g} \omega / \omega_{g}\right)^{2}} S_{0}
$$

in which the spectral parameters are $S_{0}=142.75 \mathrm{~m}^{2} / \mathrm{s}^{3}, \omega_{g}=19.07 \mathrm{~s}^{-1}$ and $\zeta_{g}=0.544$.

The PSD and variance responses of the mean valued model (MVM) were compared with those of the nonparametric probabilistic model (NPM) for the above mentioned excitations. The frequency domain was restricted to $\omega \in[0,100] \mathrm{s}^{-1}$, and the frequency interval was $0.2 \mathrm{~s}^{-1}$. The probability density function of the random system matrices are characterized by the dimension of the matrices, their mean values and a scalar parameter defining their overall randomness $[3,15,16]$. In this paper, the dispersion parameters are discussed in detail for the mean values of the random mass, damping and stiffness matrices with a certain dimension. For MVMs, the motion Eq. (11) were solved directly by means of PEM. For NPMs, the motion Eq. (10) were solved for a few dispersion parameters and the structural responses were computed by PEM combined with the Monte Carlo method. The observed DOF was the horizontal y-direction displacement of node 20. 


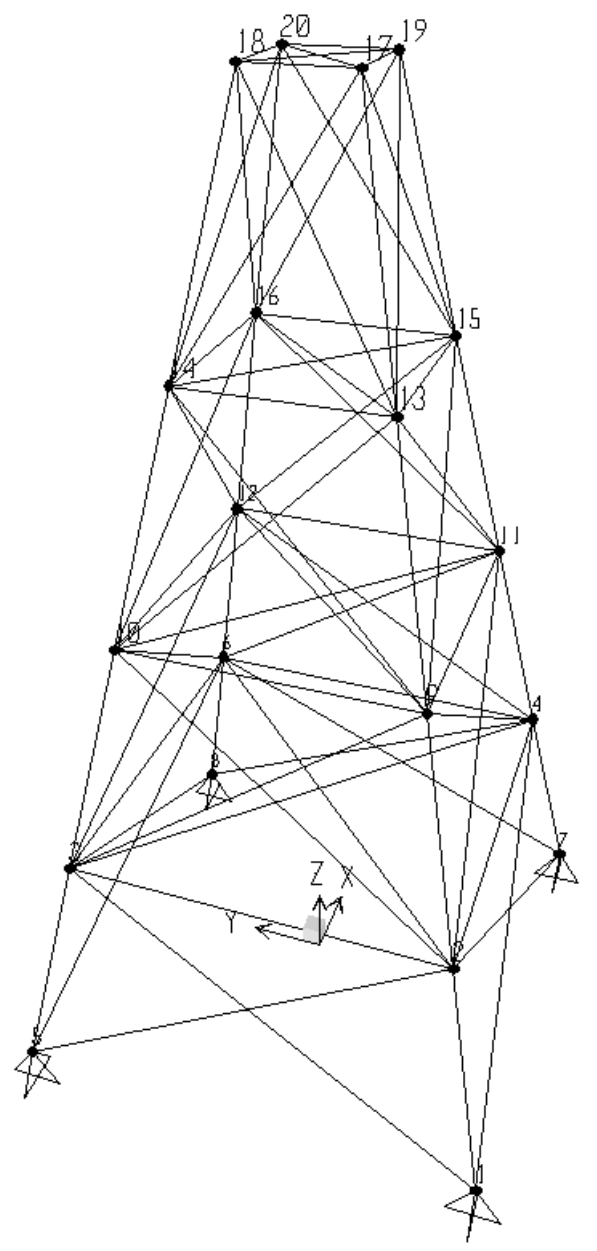

Fig. 1. 72-bar spatial truss.

\subsection{Convergence of random responses for the non-parametric probabilistic systems}

In order to investigate the convergence of dynamic responses of non-parametric probabilistic systems subjected to given stationary random excitations, two groups of dispersion parameters, i.e. $\delta_{M}=\delta_{C}=\delta_{K}=\delta$ with $\delta=$ 0.03 and $\delta=0.08$, were used for the Monte Carlo numerical simulations with samples gradually increasing to 5000 . Figure 2 demonstrates the convergence of norm $Q$, see Eq. (23), with respect to the sample numbers. It can be seen that convergence was achieved when the sample number is greater than 2500 . This effort was acceptable because the CPU time used for 5000 samples was only 896s when a Pentium (R) D personal computer with main frequency of $3.2 \mathrm{GHz}$ and a 1 Gbyte memory was used.

\subsection{Confidence region of random responses for the non-parametric probabilistic systems}

Retaining $\delta_{M}=\delta_{C}=\delta_{K}=\delta$ with $\delta=0.03$ or $\delta=0.08$, and computing the PSD and variances of the nonparametric probabilistic system subjected to filtered white noise excitations by using Eqs (20) and (21) respectively, with 5000 samples and confidence coefficient $\gamma=0.95$, the confidence region of random responses can be completed according to Section 3.3. Figure 3 gives the PSD confidence region for the dispersion parameters 0.03 and 0.08 . It can be seen that the confidence region of the PSD response with $\delta=0.03$ is less than that with $\delta=0.08$. Table 1 gives the computed variance confidence region, which shows that the region for $\delta=0.08$ is bigger than that for $\delta=0.03$. 
Table 1

$\mathrm{Y}_{20}$-variance confidence region of the stochastic system $\left(10^{-2} \mathrm{~m}^{2}\right)$

\begin{tabular}{ccccc}
\hline Dispersion parameters & \multicolumn{3}{c}{ Variance of NPM } & Variance of MVM \\
\cline { 2 - 4 } & $\bar{\theta}$ & $\theta_{1}$ & $\theta_{2}$ & \\
\hline$\delta=0.03$ & 2.555 & 2.414 & 2.696 & 2.552 \\
$\delta=0.08$ & 2.580 & 2.196 & 2.965 & 2.552 \\
\hline
\end{tabular}

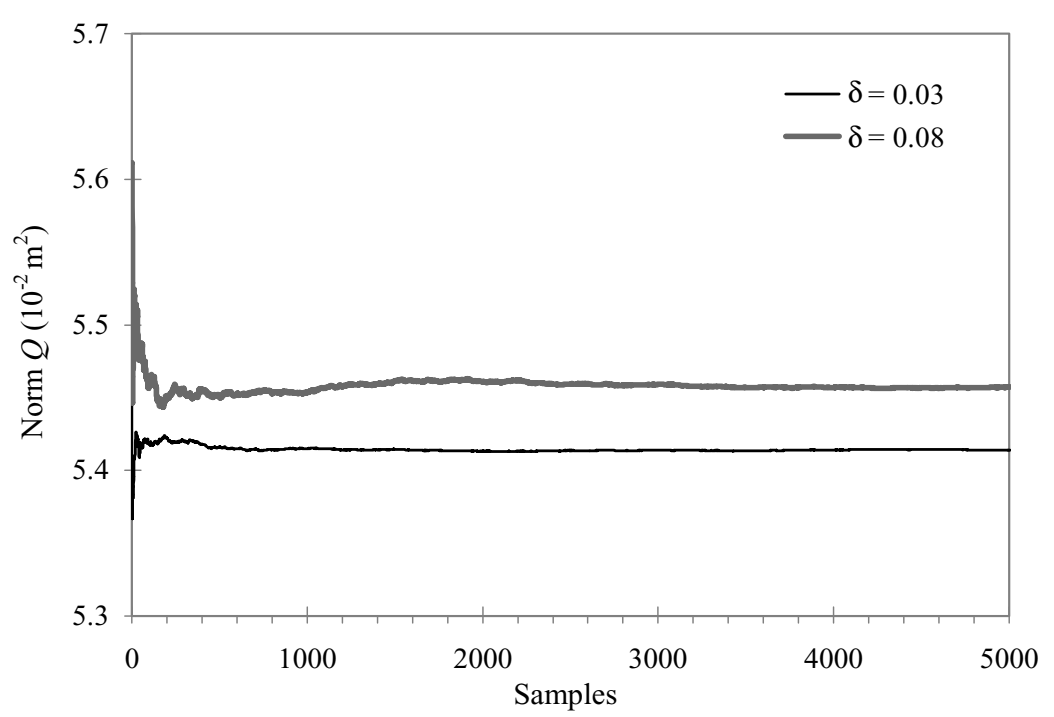

Fig. 2. Convergence analysis of $\mathrm{Y}_{20}$.

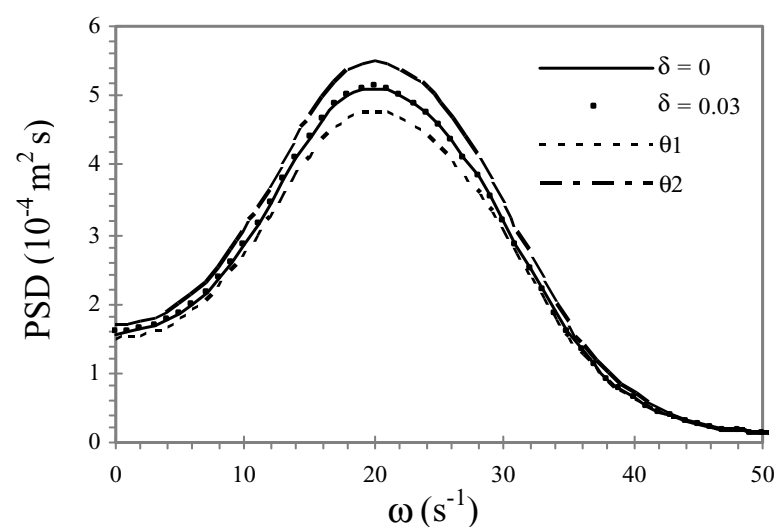

(a)

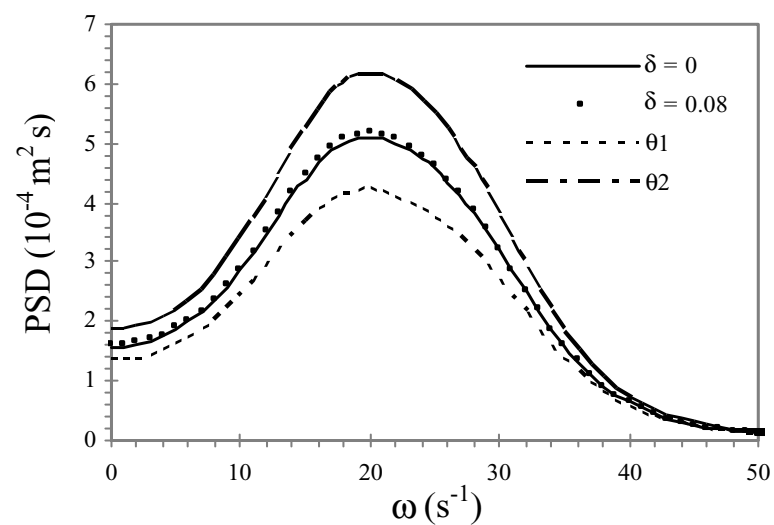

(b)

Fig. 3. $\mathrm{Y}_{20}$-PSD confidence region for dispersion parameters $\delta_{M}=\delta_{C}=\delta_{K}=\delta$ (a) for $\delta=0.03$ and; (b) for $\delta=0.08$.

Figure 3 and Table 1 also give the computed variance responses of the mean valued model $\left(\delta_{M}=\delta_{C}=\delta_{K}=\delta=\right.$ 0 ). Clearly, for a stochastic dynamical system with $\delta=0.03$ or $\delta=0.08$, the responses based on the two models are very close.

\subsection{Sensitivity analysis of random responses for non-parametric probabilistic systems}

Sensitivity analyses of $\mathrm{Y}_{20}$ for the non-parametric probabilistic systems subjected to stationary random excitations were carried out when the stochastic mass, damping and stiffness matrices varied independently with the corre- 


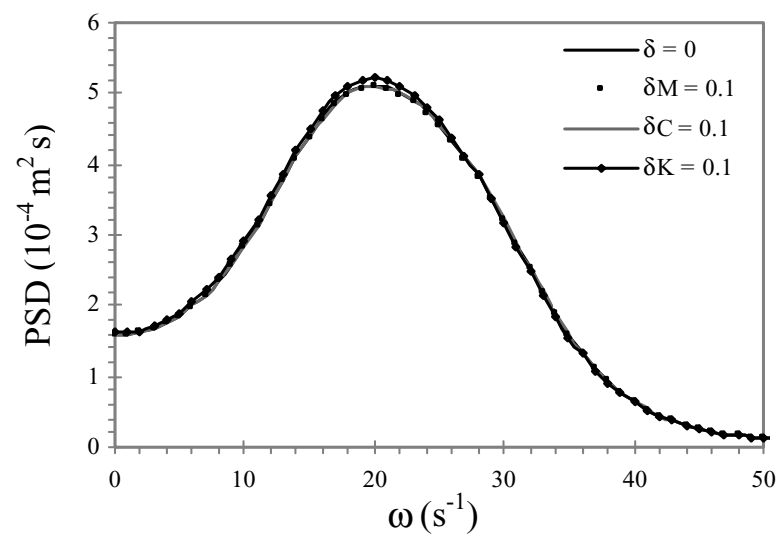

(a)

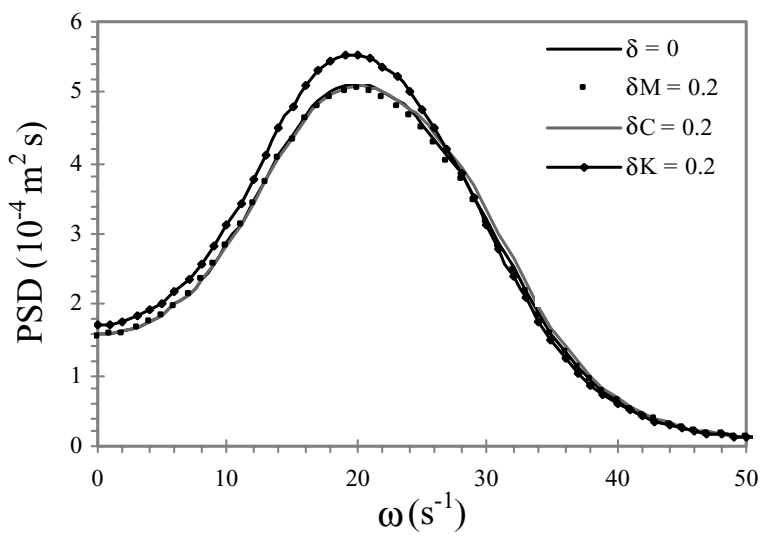

(b)

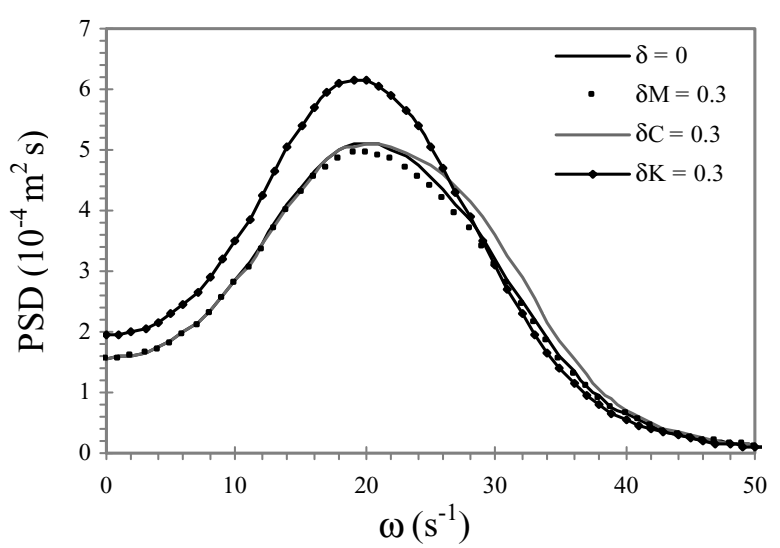

(c)

Fig. 4. PSD curves of $\mathrm{Y}_{20}$ for independently varying stochastic matrices with dispersion parameters: (a) 0.1 ; (b) 0.2 ; and (c) 0.3 .

sponding dispersion parameters. 5000 samples were used for all Monte-Carlo simulations. Figure 4(a) shows the variation of the random response PSD sensitivities for this stochastic dynamic system with the dispersion parameter combinations $\left(\delta_{M}=0.1, \delta_{C}=\delta_{K}=0\right),\left(\delta_{C}=0.1, \delta_{M}=\delta_{K}=0\right)$ and $\left(\delta_{K}=0.1, \delta_{M}=\delta_{C}=0\right)$. It can be seen that the response PSDs are sensitive to the stiffness dispersion parameter $\delta_{K}$, but not so sensitive to the mass and damping dispersion parameters $\delta_{M}$ and $\delta_{C}$. Figure 4(b) gives the corresponding results for $\left(\delta_{M}=0.2, \delta_{C}=\delta_{K}=\right.$ $0),\left(\delta_{C}=0.2, \delta_{M}=\delta_{K}=0\right)$ and $\left(\delta_{K}=0.2, \delta_{M}=\delta_{C}=0\right)$. Figure 4(c) gives the corresponding results for $\left(\delta_{M}=\right.$ $\left.0.3, \delta_{C}=\delta_{K}=0\right),\left(\delta_{C}=0.3, \delta_{M}=\delta_{K}=0\right)$ and $\left(\delta_{K}=0.3, \delta_{M}=\delta_{C}=0\right)$. Figures 4(b) and 4(c) show even more clearly that the response PSDs are particularly sensitive to $\delta_{K}$, but are relatively insensitive to $\delta_{M}$ and $\delta_{C}$.

\subsection{Stability problem of the random response analysis for non-parametric probabilistic systems}

The stability problem of the computation of $\mathrm{Y}_{20}$ for the non-parametric probabilistic system subjected to stationary random excitations is investigated when the stochastic mass, damping and stiffness matrices vary independently with the corresponding dispersion parameters all restricted within the range $0 \sim 0.3$. As before, 5000 samples were used for all Monte-Carlo simulations. Figure 5 shows the PSD curves of $\mathrm{Y}_{20}$ when the stochastic mass, damping and stiffness matrices vary independently with dispersion parameters $\delta=0,0.05,0.1,0.15,0.2,0.25$ and 0.3 . It can be seen from those figures that when the dispersion parameters $\delta$ decrease from 0.3 down to zero, the mean values of the PSD responses approach the responses for the mean FE model. Figure 6 gives some comparisons for the cases described above when the variance response values are normalized by the variance of MVM. Clearly, convergence can still be observed for all cases as $\delta$ approaches zero. 


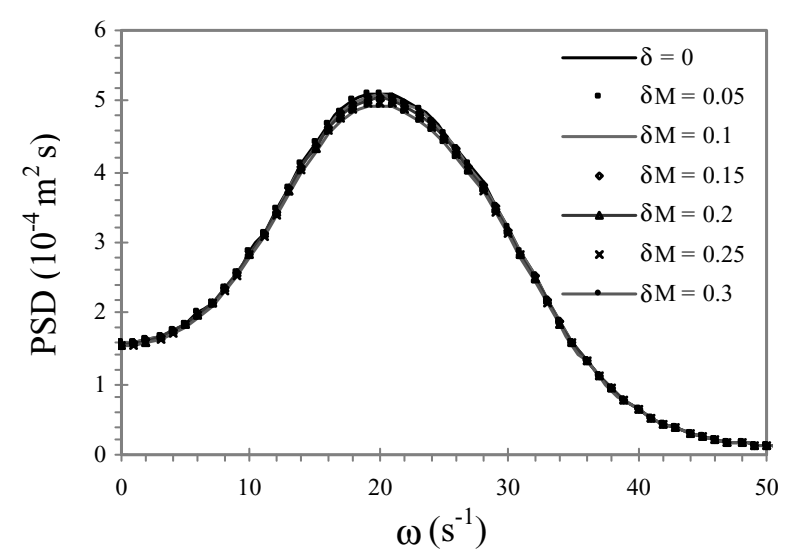

(a)

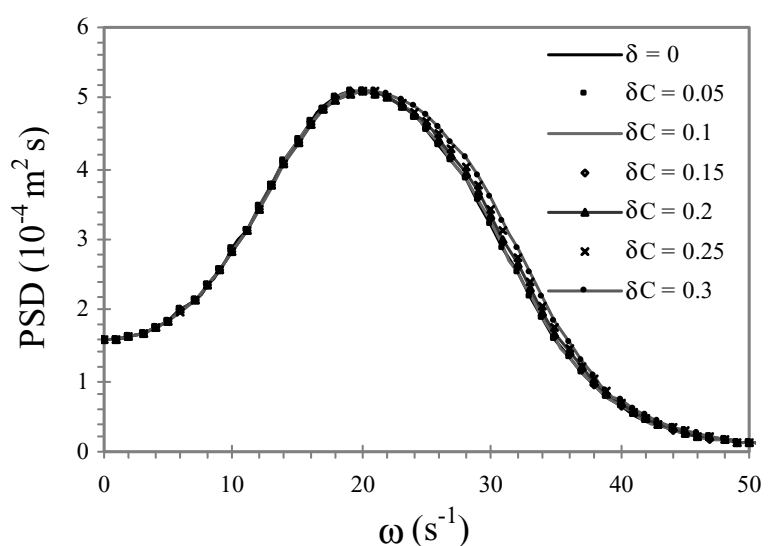

(b)

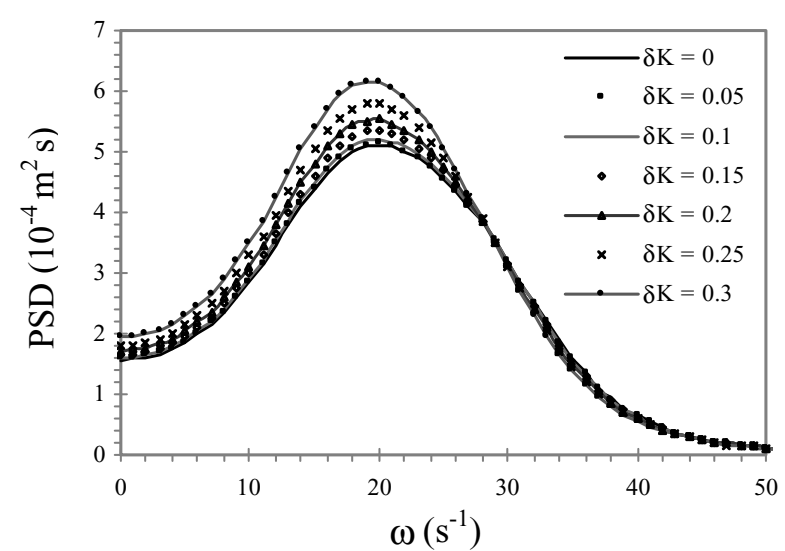

(c)

Fig. 5. PSD curves of $\mathrm{Y}_{20}$ for independently varying stochastic matrices for: (a) mass; (b) damping and; (c) stiffness.

\section{Conclusions}

Within the realms of probabilistic structural mechanics, it is often convenient to use a non-parametric probabilistic model as a theoretical representation of structure uncertainty. This has been used extremely successfully when simulating complex and uncertain structural dynamics systems, such as aircraft, offshore platforms, long-span bridges etc., which usually operate in a random load environment. The prediction of random responses for non-parametric probability systems is of great importance.

In this paper, an efficient method for analyzing a non-parametric uncertain system subjected to stationary random excitation is proposed. The random uncertainties associated with the mass, damping and stiffness matrices in a finite element model are first replaced by random matrices and then a highly efficient, pseudo excitation method for the dynamic response analysis of non-parametric probability systems subjected to stationary random loads is developed. Numerical simulations show that the dynamic responses obtained from a mean finite element model are quite different to those obtained using a random matrix model.

The sensitivity and stability of random responses for non-parametric probabilistic systems are also discussed. At present, there are still many difficulties to be aware of when undertaking a dynamic analysis using a non-parametric probability model. For example, since the actual finite element model of a complex structure may have ten thousand or more degrees of freedom, the corresponding random matrix model is generally established in modal coordinates. This has the potential to lead to large errors between the models. More generally, the range of precision that might be expected needs substantial further research. In addition to this, the analysis of a non-parametric probability system 


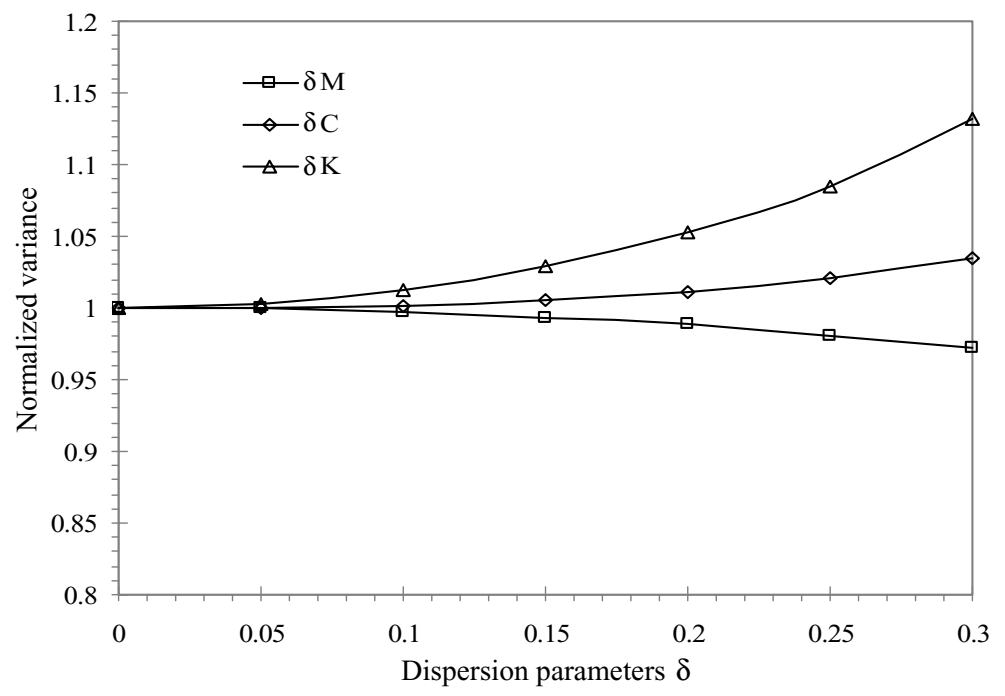

Fig. 6. Variance of $\mathrm{Y}_{20}$ for independently varying stochastic matrices.

under random excitations is not well developed either. It will be noted that this paper limits itself to a discussion of the structural responses under stationary random loads. However, the actual random excitation is generally non-stationary and hence the dynamic analysis of non-parametric probability systems subjected to non-stationary random excitations also requires further work.

\section{Acknowledgements}

The authors are grateful for support by Natural Science Foundation of China (Grant Nos. 50608012 and 10972048), the Laboratory of Seismic Control and Structural Safety and the Cardiff Advanced Chinese Engineering Centre.

\section{References}

[1] M. Kleiber and T.D. Hien, The stochastic finite element method, Chichester: Wiley, 1992.

[2] Y.K. Lin and G.Q. Cai, Probabilistic structural dynamics, New York: McGraw-Hill, 1995.

[3] C. Soize, A comprehensive overview of a non-parametric probabilistic approach of model uncertainties for predictive models in structural dynamics, Journal of Sound and Vibration 288(3) (2005), 623-652.

[4] C. Soize, Random matrix theory for modeling uncertainties in computational mechanics, Computer Methods in Applied Mechanics and Engineering 194(12-16) (2004), 1333-1366.

[5] A.S. Nowak and K.R. Collins, Reliability of structures, New York: McGraw-Hill, 2000.

[6] O. Ditlevsen and H.O. Madsen, Structural reliability methods, Chichester: Wiley, 2004.

[7] A. Der Kiureghian, First- and second-order reliability methods, Chapter 14 in: Engineering Design Reliability Handbook, CRC Press: Boca Raton, FL, 2005.

[8] E. Vanmarcke and M. Grigoriu, Stochastic finite element analysis of simple beams, Journal of Engineering Mechanics 109(5) (1983), 1203-1214.

[9] M. Shinozuka and G. Deodatis, Response variability of stochastic finite element systems, Journal of Engineering Mechanics 114(3) (1988), 499-519.

[10] R.G. Ghanem and P.D. Spanos, Stochastic finite elements: A spectral approach, Berlin: Springer, 1991.

[11] W.K. Liu, T. Belytschko and A. Mani, Random field finite elements, International Journal for Numerical Methods in Engineering 23(10) (2000), 1831-1845.

[12] M.L. Mehta, Random matrices, revised and enlarged, second ed., New York: Academic Press, 1991.

[13] C. Soize, A nonparametric model of random uncertainties for reduced matrix models in structural dynamics, Probabilistic Engineering Mechanics 15(3) (2000), 277-294.

[14] C. Soize, Transient responses of dynamical systems with random uncertainties, Probabilistic Engineering Mechanics 6(4) (2001), 363-372.

[15] S. Adhikari, Matrix variate distributions for probabilistic structural mechanics, AIAA Journal 45(7) (2007), 1748-1762. 
[16] S. Adhikari, Wishart random matrices in probabilistic structural mechanics, ASCE Journal of Engineering Mechanics 134(12) (2008), 1029-1044.

[17] J.H. Lin, A fast CQC algorithm of PSD matrices for random seismic responses, Computers \& Structures 44(3) (1992), $683-687$.

[18] J.H. Lin, Y. Zhao and Y.H. Zhang, Accurate and highly efficient algorithms for structural stationary/non-stationary random responses, Computer Methods in Applied Mechanics and Engineering 191(1-2) (2001), 103-111.

[19] J.H. Lin and Y.H. Zhang, Seismic random vibration of long-span structures, Chapter 30 in: Vibration and Shock Handbook, CRC Press: Boca Raton, FL, 2005.

[20] A. Papoulis, Probability, random variables, and stochastic processes. New York: McGraw Hill, 1965.

[21] A. Gupta, and D. Nagar, Matrix variate distributions. Monographs and surveys in pure and applied mathematics, London: Chapman and Hall/CRC, 2000

[22] A.M. Tulino and S. Verdú, Random matrix theory and wireless communications, Now Publishers Inc., Hanover, Mass, 2004.

[23] R.W. Clough and J. Penzien, Dynamics of structures, New York: McGraw-Hill, 1975. 

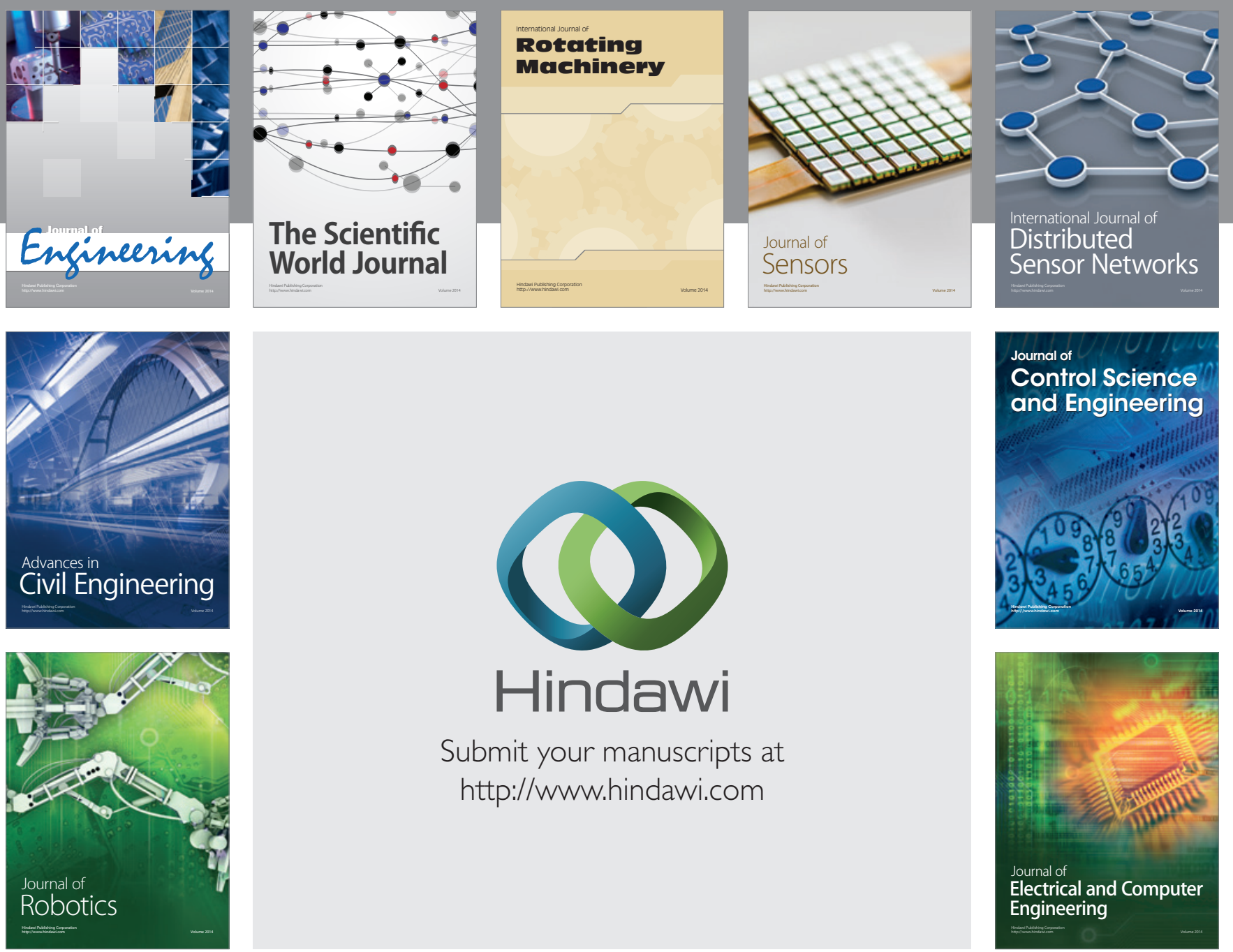

Submit your manuscripts at

http://www.hindawi.com
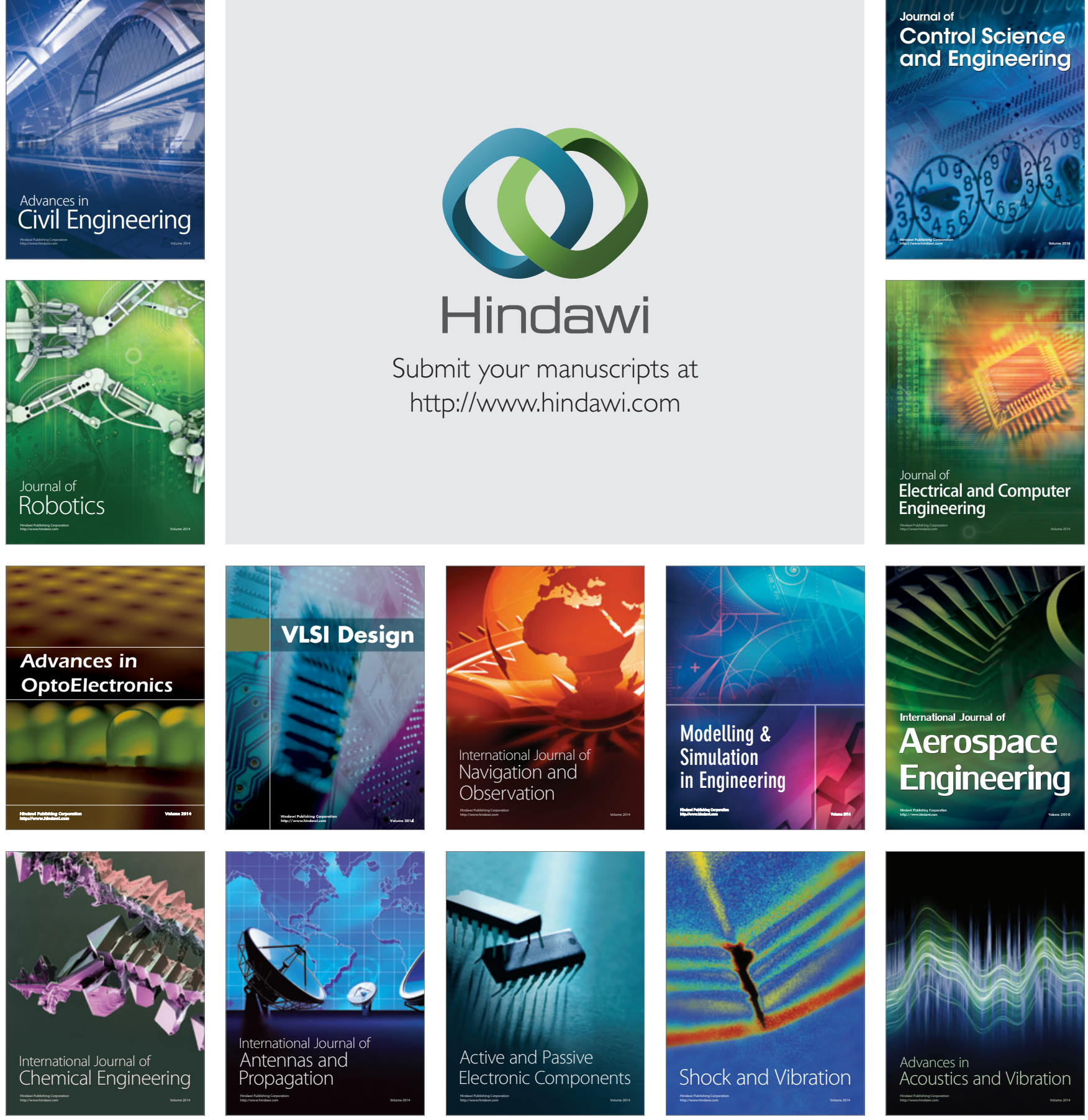